\title{
Determinant Structure of the Rational Solutions for the Painlevé IV Equation
}

\author{
KENJI KAJIWARA \\ Department of Electrical Engineering, Doshisha University, \\ Tanabe, Kyoto 610-03, Japan \\ and \\ YASUHIRO OHTA \\ Department of Applied Mathematics, Faculty of Engineering, \\ Hiroshima University, \\ 1-4-1 Kagamiyama, Higashi-Hiroshima 739, Japan
}

\begin{abstract}
Rational solutions for the Painlevé IV equation are investigated by Hirota bilinear formalism. It is shown that the solutions in one hierarchy are expressed by 3-reduced Schur functions, and those in another two hierarchies by Casorati determinant of the Hermite polynomials, or by special case of the Schur polynomials.
\end{abstract}




\section{Introduction}

It is known that the six Painlevé equations $\mathrm{P}_{\mathrm{I}}-\mathrm{P}_{\mathrm{VI}}$ are the fundamental equations in the theory of nonlinear integrable systems in wider sense, and thus their solutions are regarded as the "nonlinear version of special functions" [1]. Not only for the use of Painlevé equations in physical context, they also have many interesting mathematical structures, one of which is the structure of particular solutions. As for the algebraic solutions, it is known that some of the Painlevé equations admit rational solutions expressed by classical polynomials, e.g, Jacobi and Legendre polynomials for $\mathrm{P}_{\mathrm{VI}}$, Laguerre polynomials for $\mathrm{P}_{\mathrm{V}}$ and Hermite polynomials for $\mathrm{P}_{\mathrm{IV}}$. However, there also arise some non-classical polynomials, as pointed out by Umemura 2]. Typical example is the Yablonskii-Vorobe'v polynomials which appear in the rational solutions of $\mathrm{P}_{\mathrm{II}}$,

$$
\frac{d^{2} y}{d t^{2}}=2 y^{3}+t y+\alpha
$$

Let $T_{m}(m=0,1, \cdots)$ be polynomials generated recursively by

$$
\frac{d^{2} T_{m}}{d t^{2}} T_{m}-\left(\frac{d T_{m}}{d t}\right)^{2}=\frac{1}{4}\left(t T_{m}^{2}-T_{m+1} T_{m-1}\right), \quad T_{0}=T_{1}=1 .
$$

Then, it is known that

$$
y=\frac{d}{d t} \log \frac{T_{m+1}}{T_{m}}
$$

satisfies $\mathrm{P}_{\mathrm{II}}$ with $\alpha=-m-1$ [3, 4 . The characteristic polynomials $T_{m}$ are called the YablonskiiVorobe'v polynomials. To clarify the nature of these polynomials, it is useful to study the relation with the soliton equations. In fact, $\mathrm{P}_{\mathrm{II}}$ is derived from the similarity reduction of the modified KdV equation. This fact implies that the Yablonskii-Vorobe'v polynomials are closely related to the $\tau$ function which gives the rational solutions of the modified KdV equations. Based on this expectation, we have shown in the previous paper that Yablonskii-Vorobe'v polynomials are expressed as the specialization of the 2-reduced Schur functions [5].

In this article, we investigate the rational solutions for $\mathrm{P}_{\mathrm{IV}}$,

$$
\frac{d^{2} w}{d z^{2}}=\frac{1}{2 w}\left(\frac{d w}{d z}\right)^{2}+\frac{3}{2} w^{3}+4 z w^{2}+2\left(z^{2}-\alpha\right) w+\frac{\beta}{w},
$$

where $\alpha$ and $\beta$ are parameters. There are various simple particular solutions of physical importance [6], and it is possible to obtain solutions of "higher order" by applying the Bäcklund transformations 6,7,8,9] which map one solution to another solution of $\mathrm{P}_{\mathrm{IV}}$ with different values of parameters. As for rational solutions of $\mathrm{P}_{\mathrm{IV}}(\mathbb{4})$, it is known that there are three hierarchies of unique rational solutions[7](the name of the hierarchies are due to Ref. [10]), 
" $-\frac{1}{z}$ hierarchy":

$$
w=\frac{P_{n-1}(z)}{Q_{n}(z)}, \quad(\alpha, \beta)=\left( \pm k,-2(1+2 l+k)^{2}\right), k, l \in \mathbf{Z}, l \leq-1, k \leq-2 l,
$$

" $-2 z$ hierarchy":

$$
w=-2 z+\frac{P_{n-1}(z)}{Q_{n}(z)}, \quad(\alpha, \beta)=\left(k,-2(1+2 l+k)^{2}\right), k, l \in \mathbf{Z}, l \geq 0, k \leq-l,
$$

" $-\frac{2}{3} z$ hierarchy":

$$
w=-\frac{2}{3} z+\frac{P_{n-1}(z)}{Q_{n}(z)}, \quad(\alpha, \beta)=\left(2 k,-2\left( \pm \frac{1}{3}+2 l\right)^{2}\right),\left(2 k+1,-2\left( \pm \frac{2}{3}+2 l\right)^{2}\right), k, l \in \mathbf{Z},
$$

where $P_{m}(z)$ and $Q_{m}(z)$ are some polynomials in $z$ of degree $m$, and that there is no other rational solutions.

Lukashevich has shown that the simplest solutions in the first two hierarchies are expressed by the Hermite polynomials [1]. Okamoto has studied the Bäcklund transformations and shown that the log derivative of ratio of two-directional Wronskians of the Hermite polynomials give the solutions of $\mathrm{P}_{\mathrm{IV}}[12]$. Moreover, Murata[7] has pointed out that any solution in the $-\frac{1}{z}$ hierarchy can be transformed to a solution in the $-2 z$ hierarchy, and vice versa. These facts strongly implies that these two hierarchies have the same nature, and the solutions may be expressed by log derivative of ratio of some determinants whose entries are related with the Hermite polynomials. Those determinants are called the $\tau$ functions. We investigate these hierarchies by using the Hirota's bilinear formalism and show that all of the solutions in those hierarchies are expressed by the $\tau$ functions which is nothing but the Casorati determinants (or equivalently, the Wronskians) of the Hermite polynomials. Moreover, the $\tau$ functions are also expressible in terms of the Schur functions.

The structure of the solutions in $-\frac{2}{3} z$ hierarchy has been unknown, but Okamoto 12 has studied this hierarchy and obtained the following result.

Proposition 1.1 (Окамото) Let $Q_{m}, m \in \mathbf{Z}_{\geq 0}$, be polynomials in $x$ generated by

$$
\frac{d^{2}}{d x^{2}} \log Q_{m}+x^{2}+2 m-1=\frac{Q_{m+1} Q_{m-1}}{Q_{m}^{2}}, \quad Q_{0}=Q_{1}=1 \text {. }
$$

Then

$$
u=\frac{d}{d x} \log \frac{Q_{m+1}}{Q_{m}}-x
$$

satisfies $P_{\mathrm{IV}}$,

$$
\frac{d^{2} u}{d x^{2}}=\frac{1}{2 u}\left(\frac{d u}{d x}\right)^{2}+\frac{3}{2} u^{3}+6 x u+\frac{9}{2}\left(x^{2}-\frac{4}{3} m\right) u-\frac{1}{2 u}
$$


Similarly, let $R_{m}, m \in \mathbf{Z}_{\geq 0}$, be polynomials in $x$ generated by

$$
\frac{d^{2}}{d x^{2}} \log R_{m}+x^{2}+2 m=\frac{R_{m+1} R_{m-1}}{R_{m}^{2}}, \quad R_{0}=1, \quad R_{1}=x .
$$

Then

$$
u=\frac{d}{d x} \log \frac{R_{m+1}}{R_{m}}-x
$$

satisfies $P_{\mathrm{IV}}$,

$$
\frac{d^{2} u}{d x^{2}}=\frac{1}{2 u}\left(\frac{d u}{d x}\right)^{2}+\frac{3}{2} u^{3}+6 x u+\frac{9}{2}\left(x^{2}-\frac{4}{3}\left(m+\frac{1}{2}\right)\right) u-\frac{4}{2 u} .
$$

The characteristic polynomials $Q_{m}$ and $R_{m}$ are called the Okamoto polynomials [2]. Indeed, the solutions which are expressed by the Okamoto polynomials are special case of the $-\frac{2}{3} z$ hierarchy.

The key to understand the nature of the Okamoto polynomials lies in the relation with soliton equations, as is in the case of $\mathrm{P}_{\mathrm{II}}$. In fact, several authors have pointed out the relation between $\mathrm{P}_{\mathrm{IV}}$ and the Boussinesq equation [13, 14, 15, 16] which belongs to the 3-reduction of the KP hierarchy [17]. This implies that some solutions of $\mathrm{P}_{\mathrm{IV}}$ may be understood as the similarity reduction of the 3 -reduced KP hierarchy. We show that the solutions in the $-2 / 3 z$ hierarchy is indeed the case, namely they are expressed by the 3-reduced Schur functions.

\section{$2 \quad-\frac{2}{3} z$ Hierarchy}

Let us consider the Schur functions in $x_{1}, x_{2}, \cdots$ labelled by Young diagram $Y=\left(i_{1}, i_{2}, i_{3}, \cdots, i_{l}\right)$, $i_{1} \geq i_{2} \geq \cdots \geq i_{l} \geq 0$

$$
S_{Y}\left(x_{1}, x_{2}, \cdots\right)=\left|\begin{array}{cccc}
p_{i_{1}} & p_{i_{1}+1} & \cdots & p_{i_{1}+l-1} \\
p_{i_{2}-1} & p_{i_{2}} & \cdots & p_{i_{2}+l-2} \\
\vdots & \vdots & \ddots & \vdots \\
p_{i_{l}-l+1} & p_{i_{l}-l+2} & \cdots & p_{i_{l}}
\end{array}\right|,
$$

where $p_{k}$ 's are polynomials in $x_{1}, x_{2}, \cdots$ defined by

$$
\sum_{k=0}^{\infty} p_{k}\left(x_{1}, x_{2}, \cdots\right) \lambda^{k}=\exp \sum_{n=1}^{\infty} x_{k} \lambda^{k}, \quad p_{n}=0(n<0) .
$$

It is well known that $S_{Y}$ is a $\tau$ function of the KP hierarchy [17.

We consider the 3-reduction of the $\tau$ function, namely, we impose the condition,

$$
\frac{\partial \tau_{Y}}{\partial x_{3 k}}=0, \quad k=1,2, \cdots .
$$


To realize this condition, it is sufficient to restrict the Young diagram as

$$
Y=\left(M+2 n, M+2 n-2, \cdots, M+2, M^{2},(M-1)^{2}, \cdots, 2^{2}, 1^{2}\right),
$$

or

$$
Y=\left(N+2 n-1, N+2 n-3, \cdots, N+1, N^{2},(N-1)^{2}, \cdots, 2^{2}, 1^{2}\right) .
$$

In fact, we can easily verify that the Schur functions associated with the above Young diagrams satisfy the condition (16), noticing the relation,

$$
\frac{\partial p_{n}}{\partial x_{k}}=p_{n-k} .
$$

For notational simplicity, we rearrange the structure of the Schur polynomials associated with the Young diagrams (17) and (18) as

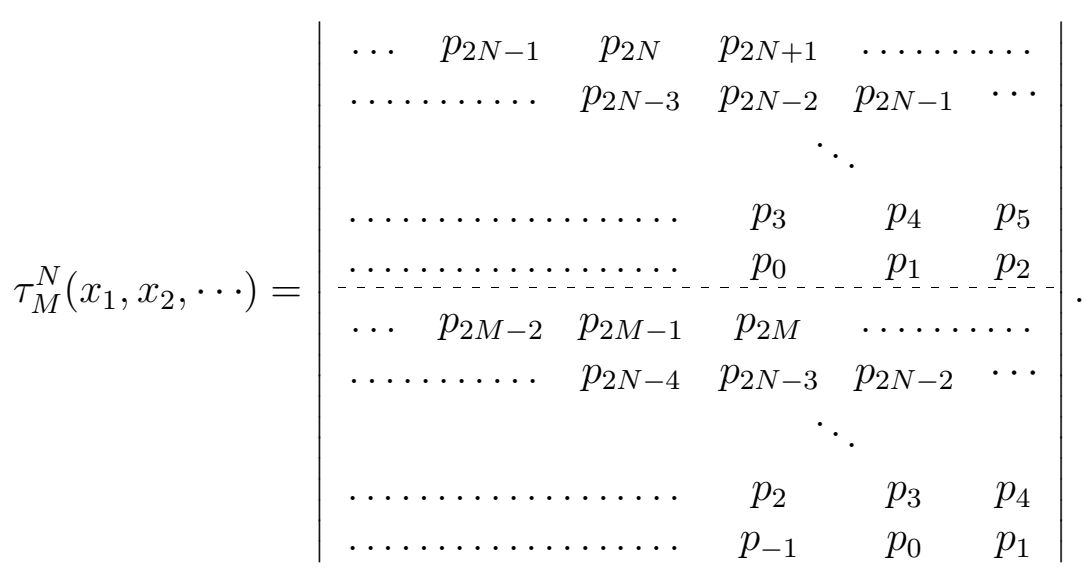

We can see the equivalence of $\tau_{M}^{N}$ and 3-reduced Schur functions in the following manner,

$$
\tau_{M}^{N}= \begin{cases}S_{\left(M+2 n, M+2 n-2, \cdots, M+2, M^{2},(M-1)^{2}, \cdots, 1^{2}\right)} & \text { for } N=M+n>M, \\ S_{\left(N+2 n-1, N+2 n-3, \cdots, N+1, N^{2},(N-1)^{2}, \cdots, 1^{2}\right)} & \text { for } N<M=N+n .\end{cases}
$$

Then, we have the following theorem:

Theorem 2.1 Let $x_{1}=x, x_{2}=\frac{1}{2}, x_{k}=0(k=3,4, \cdots)$ in $\tau_{M}^{N}$. Then

$$
w=\sqrt{\frac{2}{3}}\left\{\left(\log \frac{\tau_{M+1}^{N}}{\tau_{M}^{N}}\right)_{x}-x\right\}, \quad z=\sqrt{\frac{3}{2}} x,
$$

satisfies $P_{\mathrm{IV}}$ (田) with

$$
(\alpha, \beta)=\left(2 M-N+1,-2\left(N+\frac{2}{3}\right)^{2}\right) \quad M, N \in \mathbf{Z}_{\geq 0} .
$$

Similarly,

$$
w=\sqrt{\frac{2}{3}}\left\{\left(\log \frac{\tau_{M}^{N+1}}{\tau_{M}^{N}}\right)_{x}-x\right\}, \quad z=\sqrt{\frac{3}{2}} x,
$$


satisfies $P_{\mathrm{IV}}$ (4) with

$$
(\alpha, \beta)=\left(-M+2 N+2,-2\left(M+\frac{1}{3}\right)^{2}\right) \quad M, N \in \mathbf{Z}_{\geq 0}
$$

\section{REMARK 2.2}

The above solutions cover all of the $-\frac{2}{3} z$ hierarchy, which is easily verified by comparing the parameters (23) and (25) with (7).

Moreover, we have:

COROLlary 2.3 The Okamoto polynomials are the special case of 3-reduced Schur functions,

$$
Q_{m}=\tau_{0}^{m}=S_{(2 m, 2 m-2, \cdots, 2)}\left(x, \frac{1}{2}, 0, \cdots\right), \quad R_{m}=\tau_{m}^{0}=S_{(2 n-1,2 n-3, \cdots, 1)}\left(x, \frac{1}{2}, 0, \cdots\right) .
$$

Theorem 2.1 is proved by considering the similarity reduction of 3-reduction of the first modified KP hierarchy, namely, hierarchy of the Bäcklund transformations of the KP hierarchy. First three of the bilinear equations in this hierarchy are given by 17,

$$
\begin{gathered}
\left(D_{x_{1}}^{2}-D_{x_{2}}\right) \tau_{M+1}^{N} \cdot \tau_{M}^{N}=0 \\
\left(D_{x_{1}}^{3}+3 D_{x_{1}} D_{x_{2}}\right) \tau_{M+1}^{N} \cdot \tau_{M}^{N}=0 \\
\left(D_{x_{1}}^{4}-3 D_{x_{1}}^{2} D_{x_{2}}-6 D_{x_{2}}^{2}\right) \tau_{M+1}^{N} \cdot \tau_{M}^{N}=0
\end{gathered}
$$

where $D_{x}$ is the Hirota's bilinear differential operator defined by

$$
D_{x}^{n} f \cdot g=\left.\left(\partial_{x}-\partial_{x^{\prime}}\right)^{n} f(x) g\left(x^{\prime}\right)\right|_{x=x^{\prime}}
$$

Next, we apply the similarity reduction.

Lemma 2.4 Let $\tau_{N}^{M}=\tau_{N}^{M}\left(x_{1}, x_{2}, 0,0, \cdots\right)$. Then

$$
\partial_{x_{2}} \tau_{M}^{N}=\frac{1}{2 x_{2}}\left(\left(M^{2}-M N+N^{2}+N\right) \tau_{M}^{N}-x_{1} \partial_{x_{1}} \tau_{M}^{N}\right)
$$

Proof: Notice that $p_{k}$, the entry of $\tau_{M}^{N}$, is a homogeneous polynomial in $\left(x_{1}, x_{2}, x_{3}, \cdots\right)$ with degree $k$ if we define the degree of $x_{k}$ as $k$. Putting $x_{k}=0(k \geq 3)$, then $\tau_{M}^{N}$ is a homogeneous polynomial in $x_{1}$ and $x_{2}$ of degree $M^{2}-M N+N^{2}+N$. Thus, if we set

$$
f_{M}^{N}=\frac{1}{x_{2}^{\left(M^{2}-M N+N^{2}+N\right) / 2}} \tau_{M}^{N},
$$


then $f_{M}^{N}$ depends only on $t=\frac{x_{1}}{x_{2}^{1 / 2}}$. This implies

$$
\begin{gathered}
\partial_{x_{2}} f_{M}^{N}=\frac{\partial t}{\partial x_{2}} \frac{d}{d t} f_{M}^{N}=-\frac{1}{2} \frac{x_{1}}{x_{2}^{3 / 2}} \frac{d}{d t} f_{M}^{N} \\
\partial_{x_{1}} f_{M}^{N}=\frac{\partial t}{\partial x_{1}} \frac{d}{d t} f_{M}^{N}=\frac{1}{x_{2}^{1 / 2}} \frac{d}{d t} f_{M}^{N}
\end{gathered}
$$

which yields

$$
-2 x_{2} \partial_{x_{2}} f_{M}^{N}=x_{1} \partial_{x_{1}} f_{M}^{N}
$$

Rewriting eq.(35) in terms of $\tau_{M}^{N}$, we get eq. (31).

By using Lemma 2.4, all the $x_{2}$ derivatives in eqs.(27)-(29) are rewritten in terms of $x_{1}$ derivatives as

$$
\begin{gathered}
\left(D_{x_{1}}^{2}+\frac{x_{1}}{2 x_{2}} D_{x_{1}}-\frac{1}{2 x_{2}}(2 M-N+1)\right) \tau_{M+1}^{N} \cdot \tau_{M}^{N}=0 \\
\left(D_{x_{1}}^{3}-\frac{3}{2 x_{2}}\left(-x_{1} D_{x_{1}}^{2}+(2 M-N+1) D_{x_{1}}+\partial_{x_{1}}\right)\right) \tau_{M+1}^{N} \cdot \tau_{M}^{N}=0 \\
{\left[D_{x_{1}}^{4}-\frac{3}{2 x_{2}}\left(-x_{1} D_{x_{1}}^{3}+(2 M-N+1) D_{x_{1}}^{2}-2 \partial_{x_{1}} D_{x_{1}}\right)\right.} \\
\left.-\frac{6}{4 x_{2}^{2}}\left(x_{1}^{2} D_{x_{1}}^{2}-2 x_{1}(2 M-N+1) D_{x_{1}}+3 x_{1} \partial_{x_{1}}-\left(3 N^{2}+4 N+1\right)\right)\right] \tau_{M+1}^{N} \cdot \tau_{M}^{N}=0
\end{gathered}
$$

respectively, where

$$
\begin{gathered}
\partial_{x_{1}} D_{x_{1}} f \cdot g=f_{x_{1} x_{1}} g-f g_{x_{1} x_{1}}, \\
\partial_{x_{1}} f \cdot g=\partial_{x_{1}}(f g)=f_{x_{1}} g+f g_{x_{1}} .
\end{gathered}
$$

Putting $x_{1}=x$ and $x_{2}=\frac{1}{2}$, we have:

Proposition $2.5 \tau_{M}^{N}=\tau_{M}^{N}\left(x, \frac{1}{2}, 0, \cdots\right)$ and $\tau_{M+1}^{N}=\tau_{M+1}^{N}\left(x, \frac{1}{2}, 0, \cdots\right)$ satisfy the following bilinear equations,

$$
\begin{gathered}
\left(D_{x}^{2}+x D_{x}-(2 M-N+1)\right) \tau_{M+1}^{N} \cdot \tau_{M}^{N}=0 \\
\left(D_{x}^{3}-3\left(-x D_{x}^{2}+(2 M-N+1) D_{x}+\partial_{x}\right)\right) \tau_{M+1}^{N} \cdot \tau_{M}^{N}=0 \\
{\left[D_{x}^{4}+3 x D_{x}^{3}-3\left\{2 x^{2}+(2 M-N+1)\right\} D_{x}^{2}+12 x(2 M-N+1) D_{x}\right.} \\
\left.-6\left(-\partial_{x} D_{x}+3 x \partial_{1}-\left(3 N^{2}+4 N+1\right)\right)\right] \tau_{M}^{N} \cdot \tau_{M+1}^{N}=0 .
\end{gathered}
$$


Now the proof of Theorem 2.1 is strait-forward. Dividing eqs.(41)-(43) by $\tau_{M}^{N} \tau_{M+1}^{N}$ and using the formulas[19],

$$
\begin{aligned}
\frac{D_{x} \tau_{M}^{N} \cdot \tau_{M+1}^{N}}{\tau_{M}^{N} \tau_{M+1}^{N}} & =\phi_{x} \\
\frac{D_{x}^{2} \tau_{M}^{N} \cdot \tau_{M+1}^{N}}{\tau_{M}^{N} \tau_{M+1}^{N}} & =\rho_{x x}+\phi_{x}^{2} \\
\frac{D_{x}^{3} \tau_{M}^{N} \cdot \tau_{M+1}^{N}}{\tau_{M}^{N} \tau_{M+1}^{N}} & =\phi_{x x x}+3 \phi_{x} \rho_{x x}+\phi_{x}^{3} \\
\frac{D_{x}^{4} \tau_{M}^{N} \cdot \tau_{M+1}^{N}}{\tau_{M}^{N} \tau_{M+1}^{N}} & =\rho_{x x x x}+4 \phi_{x} \phi_{x x x}+3 \rho_{x x}^{2}+6 \phi_{x}^{2} \rho_{x x}+\phi_{x}^{4}, \\
\frac{\partial_{x} D_{x} \tau_{M}^{N} \cdot \tau_{M+1}^{N}}{\tau_{M}^{N} \tau_{M+1}^{N}} & =\phi_{x x}+\phi_{x} \rho_{x},
\end{aligned}
$$

where $\rho=\log \left(\tau_{M}^{N} \tau_{M+1}^{N}\right)$ and $\phi=\log \left(\tau_{M+1}^{N} / \tau_{M}^{N}\right)$, we get

$$
\begin{gathered}
\rho_{x x}+\phi_{x}^{2}+x \phi_{x}-(2 M-N+1)=0, \\
\phi_{x x x}+3 \phi_{x} \rho_{x x}+\phi_{x}^{3}-3 x\left(\rho_{x x}+\phi_{x}^{2}\right)+3(2 M-N+1) \phi_{x}-3 \rho_{x}=0, \\
\rho_{x x x x}+4 \phi_{x} \phi_{x x x}+3 \rho_{x x}^{2}+6 \phi_{x}^{2} \rho_{x x}+\phi_{x}^{4} \\
+3 x\left(\phi_{x x x}+3 \phi_{x} \rho_{x x}+\phi_{x}^{3}\right)-3\left(2 M-N+1+2 x^{2}\right)\left(\rho_{x x}+\phi_{x}^{2}\right) \\
+12 x(2 M-N+1) \phi_{x}+6\left(\phi_{x x}+\phi_{x} \rho_{x}\right)-18 x \rho_{x}+6\left(3 N^{2}+4 N+1\right)=0,
\end{gathered}
$$

respectively. Eliminating $\rho$ and putting $u=\phi_{x}-x$, we obtain $\mathrm{P}_{\mathrm{IV}}$,

$$
\frac{d^{2} u}{d x^{2}}=\frac{1}{2 u}\left(\frac{d u}{d x}\right)^{2}+\frac{3}{2} u^{3}+6 x u^{2}+\left(\frac{9}{2} x^{2}-3 a\right) u-\frac{3 b+1}{2 u}
$$

where,

$$
a=2 M-N+1, \quad b=3 N^{2}+4 N+1 .
$$

This gives the half of the Theorem 2.1. Another half is proved in similar manner by starting from the bilinear equations,

$$
\begin{gathered}
\left(D_{x_{1}}^{2}-D_{x_{2}}\right) \tau_{M}^{N+1} \cdot \tau_{M}^{N}=0, \\
\left(D_{x_{1}}^{3}+3 D_{x_{1}} D_{x_{2}}\right) \tau_{M}^{N+1} \cdot \tau_{M}^{N}=0, \\
\left(D_{x_{1}}^{4}-3 D_{x_{1}}^{2} D_{x_{2}}-6 D_{x_{2}}^{2}\right) \tau_{M}^{N+1} \cdot \tau_{M}^{N}=0,
\end{gathered}
$$

from which we get eq. (52) for $u=\left(\log \tau_{M}^{N+1} / \tau_{M}^{N}\right)-x$ with $a=-M+2 N+2$ and $b=3 M^{2}+2 M$. This completes the proof of Theorem 2.1. 


\section{$3-\frac{1}{z}$ and $-2 z$ Hierarchies}

\section{$3.1 \tau$ Function}

As mentioned in the introduction, solutions in the $-\frac{1}{z}$ and $-2 z$ hierarchies can be transformed each other by Bäcklund transformations. From the view of $\tau$ functions, this fact suggests that their $\tau$ functions are the same, and only the relation between the $\tau$ functions and the dependent variable of $\mathrm{P}_{\mathrm{IV}}$ is different.

Definition 3.1 Let $H_{n}$ and $\hat{H}_{n}, n=0,1,2 \cdots$, be polynomials in $x$ defined by

$$
\begin{aligned}
\sum_{n=0}^{\infty} \frac{1}{n !} H_{n} \lambda^{n} & =\exp \left(x \lambda-\frac{1}{2} \lambda^{2}\right), \\
\sum_{n=0}^{\infty} \frac{1}{n !} \hat{H}_{n} \lambda^{n} & =\exp \left(x \lambda+\frac{1}{2} \lambda^{2}\right),
\end{aligned}
$$

respectively. Then we define the $\tau$ functions $\tau_{N}^{n}$ and $\hat{\tau}_{N}^{n}$ to be $N \times N$ determinants given by

$$
\begin{aligned}
\tau_{N}^{n} & =\left|\begin{array}{cccc}
H_{n} & H_{n+1} & \cdots & H_{n+N-1} \\
H_{n+1} & H_{n+2} & \cdots & H_{n+N} \\
\vdots & \vdots & \ddots & \vdots \\
H_{n+N-1} & H_{n+N} & \cdots & H_{n+2 N-2}
\end{array}\right|, \\
\hat{\tau}_{N}^{n} & =\left|\begin{array}{cccc}
\hat{H}_{n} & \hat{H}_{n+1} & \cdots & \hat{H}_{n+N-1} \\
\hat{H}_{n+1} & \hat{H}_{n+2} & \cdots & \hat{H}_{n+N} \\
\vdots & \vdots & \ddots & \vdots \\
\hat{H}_{n+N-1} & \hat{H}_{n+N} & \cdots & \hat{H}_{n+2 N-2}
\end{array}\right|,
\end{aligned}
$$

respectively.

Then, the solutions in the $-\frac{1}{z}$ hierarchy are expressed as follows:

THEOREM 3.2

$$
w=-\sqrt{2}\left(\log \frac{\tau_{N+1}^{n}}{\tau_{N}^{n}}\right)_{x}, \quad z=\frac{1}{\sqrt{2}} x,
$$

give rational solutions of $P_{\mathrm{IV}}($ 因) with

$$
(\alpha, \beta)=\left(-(n+2 N+1),-2 n^{2}\right), \quad n, N \in \mathbf{Z}, \quad n \geq 1, \quad N \geq 0 .
$$

Moreover,

$$
w=\sqrt{2}\left(\log \frac{\hat{\tau}_{N+1}^{n}}{\hat{\tau}_{N}^{n}}\right)_{x}, \quad z=\frac{1}{\sqrt{2}} x,
$$

give rational solutions of $P_{\mathrm{IV}}($ 因 with

$$
(\alpha, \beta)=\left(n+2 N+1,-2 n^{2}\right), \quad n, N \in \mathbf{Z}, \quad n \geq 1, \quad N \geq 0 .
$$


Here, several remarks are in order.

\section{REMARK 3.3}

1. Parameterization eq.(62) is equivalent to eq.(50) with $\alpha=-k$ if we put $k=n+2 N+1$ and $l=-(N+1)$. Moreover, eq.(64) is equivalent to eq.(5) with $\alpha=k$ if we put $k=n+2 N+1$ and $l=-(N+1)$. Hence the solutions in Theorem 3.2 cover all of $-\frac{1}{z}$ hierarchy.

2. As shown in the proof, $n$ do not have to be necessarily integers and hence $H_{n}$ can be the Hermite-Weber functions. In fact, we need only the recursion relations,

$$
\frac{d}{d x} H_{n}-x H_{n}=-H_{n+1}, \quad \frac{d}{d x} H_{n}=n H_{n-1} .
$$

Moreover, $\tau$ function admits several expressions.

\section{REMARK 3.4}

1. It is possible to express the solutions in terms of the Wronskian, since we have the relation,

$$
\begin{aligned}
& \left|\begin{array}{cccc}
H_{n} & H_{n+1} & \cdots & H_{n+N-1} \\
H_{n+1} & H_{n+2} & \cdots & H_{n+N} \\
\vdots & \vdots & \ddots & \vdots \\
H_{n+N-1} & H_{n+N} & \cdots & H_{n+2 N-2}
\end{array}\right| \\
& =\prod_{k=1}^{N-1}(n+k)^{k}\left|\begin{array}{cccc}
H_{n+N-1} & \frac{d}{d x} H_{n+N-1} & \cdots & \left(\frac{d}{d x}\right)^{N-1} H_{n+N-1} \\
\frac{d}{d x} H_{n+N-1} & \frac{d^{2}}{d x^{2}} H_{n+N-1} & \cdots & \left(\frac{d}{d x}\right)^{N} H_{n+N-1} \\
\vdots & \vdots & \ddots & \vdots \\
\left(\frac{d}{d x}\right)^{N-1} H_{n+N-1} & \left(\frac{d}{d x}\right)^{N} H_{n+N-1} & \cdots & \left(\frac{d}{d x}\right)^{2 N-2} H_{n+N-1}
\end{array}\right|,
\end{aligned}
$$

which can be verified by using eq.(65).

2. As is obvious from the Wronskian expression eq.([66), $\tau_{N}^{0}$ gives only a constant, which yields 0 solution of $\mathrm{P}_{\mathrm{IV}}$. Thus only the cases of $n \geq 1$ give nontrivial solutions.

3. $\tau$ functions are also expressed by the Schur functions. In fact we have, for example,

$$
\hat{\tau}_{N}^{n}=\frac{(n+N-1)^{!}}{(n-1)^{!}}(-1)^{N(N-1) / 2} S_{\left(n^{N}\right)}\left(x, \frac{1}{2}, 0, \cdots\right),
$$

where,

$$
k^{!}=k !^{k}(k-1) !^{(k-1)} \cdots 2 !^{2} 1 !^{1},
$$

which can be verified by noticing

$$
\hat{H}_{n}=\frac{1}{n !} p_{n}\left(x, \frac{1}{2}, 0, \cdots\right),
$$

and eq.(66). 
For the solutions in the $-2 z$ hierarchy, the following expression is valid.

THEOREM 3.5

$$
w=\sqrt{2}\left\{\left(\log \frac{\tau_{N+1}^{n}}{\tau_{N}^{n+1}}\right)_{x}-x\right\}, \quad z=\frac{1}{\sqrt{2}} x,
$$

give rational solutions of $P_{\mathrm{IV}}($ 因) with

$$
(\alpha, \beta)=\left(N-n,-2(n+N+1)^{2}\right), \quad n, N \in \mathbf{Z}, \quad n \geq 0, \quad N \geq 0 .
$$

\section{REMARK 3.6}

1. Parameterization eq. (70) is equivalent to eq.(6), if we put $k=N-n, l=N$. Hence the solutions in Theorem 3.5 covers all the solutions in the $-2 z$ hierarchy.

2. Another $\tau$ function $\hat{\tau}_{N}^{n}(\sqrt[60)]{ }$ can give the solution of the same type for $\mathrm{P}_{\mathrm{IV}}$. In fact, we can show that

$$
\hat{w}=-\sqrt{2}\left\{\log \left(\frac{\hat{\tau}_{N+1}^{n}}{\hat{\tau}_{N}^{n+1}}\right)_{x}-x\right\}, \quad z=\frac{1}{\sqrt{2}} x,
$$

satisfies $\mathrm{P}_{\mathrm{IV}}$ with the parameters $(\alpha, \beta)=\left(n-N,-2(n+N+1)^{2}\right)$. This parameterization is also equivalent to eq.(6), if we put $k=n-N, l=n$. However, the uniqueness of the rational solutions [7, 18] implies that they give the same solution as given in Theorem 3.5. In fact, we can check that $w$ and $\hat{w}$ are the same if we exchange $n$ and $N$.

\subsection{Proof of Theorems}

In this section, we give the proof of the Theorems 3.2 and 3.5. The first half of Theorem 3.2 is a direct consequence of the following proposition.

Proposition $3.7 \tau_{N}^{n}$ satisfies the following bilinear equations:

$$
\begin{gathered}
\left(D_{x}^{2}-x D_{x}+n\right) \tau_{N+1}^{n} \cdot \tau_{N}^{n}=0 \\
\left(D_{x}^{3}-6 x D_{x}^{2}+\left(5 x^{2}+n-4 N-2\right) D_{x}+\frac{d}{d x}-5 n x\right) \tau_{N+1}^{n} \cdot \tau_{N}^{n}=0 \\
\left(D_{x}^{4}-4 x D_{x}^{3}+\left(11 x^{2}-8 n-28 N-14\right) D_{x}^{2}-2 \frac{d}{d x} D_{x}-2 x\left(4 x^{2}-3 n-20 N-10\right) D_{x}\right. \\
\left.-3 x \frac{d}{d x}+8 n x^{2}-n(9 n+28 N+14)\right) \tau_{N+1}^{n} \cdot \tau_{N}^{n}=0 .
\end{gathered}
$$

The second half of Theorem 3.2 is derived from the following bilinear equations: 
Proposition $3.8 \hat{\tau}_{N}^{n}$ satisfies the following bilinear equations.

$$
\begin{gathered}
\left(D_{x}^{2}+x D_{x}-n\right) \hat{\tau}_{N+1}^{n} \cdot \hat{\tau}_{N}^{n}=0 \\
\left(D_{x}^{3}+6 x D_{x}^{2}+\left(5 x^{2}-n+4 N+2\right) D_{x}-\frac{d}{d x}-5 n x\right) \hat{\tau}_{N+1}^{n} \cdot \hat{\tau}_{N}^{n}=0 \\
\left(D_{x}^{4}+4 x D_{x}^{3}+\left(11 x^{2}+8 n+28 N+14\right) D_{x}^{2}+2 \frac{d}{d x} D_{x}+2 x\left(4 x^{2}+3 n+20 N+10\right) D_{x}\right. \\
\left.-3 x \frac{d}{d x}-8 n x^{2}-n(9 n+28 N+14)\right) \hat{\tau}_{N+1}^{n} \cdot \hat{\tau}_{N}^{n}=0 .
\end{gathered}
$$

Finally, Theorem 3.5 is obtained from the following bilinear equations:

Proposition $3.9 \tau_{N}^{n}$ satisfies the following bilinear equations.

$$
\begin{gathered}
\left(D_{x}^{2}-x D_{x}+n-N\right) \tau_{N+1}^{n} \cdot \tau_{N}^{n+1}=0 \\
\left(D_{x}^{3}-6 x D_{x}^{2}+\left(5 x^{2}+n-N\right) D_{x}+\frac{d}{d x}-5(n-N) x\right) \tau_{N+1}^{n} \cdot \tau_{N}^{n+1}=0 \\
\left(D_{x}^{4}-4 x D_{x}^{3}+\left(11 x^{2}-8 n-28 N-18\right) D_{x}^{2}+2 \frac{d}{d x} D_{x}-2 x\left(4 x^{2}-3 n-15 N-9\right) D_{x}\right. \\
\left.-x \frac{d}{d x}+8(n-N) x^{2}-\left(9 n^{2}+10 N n+14 n-27 N^{2}-22 N\right)\right) \tau_{N+1}^{n} \cdot \tau_{N}^{n+1}=0
\end{gathered}
$$

To prove the Propositions 3.7 3.9, we first introduce the notation $\tau_{N Y}^{n}$ :

Definition 3.10 Let $Y=\left(i_{1}, i_{2}, \cdots, i_{h}\right)$ be a Young diagram. Then we define an $N \times N$ determinant $\tau_{N Y}^{n}$ by

$$
\tau_{N Y}^{n}=\left|\begin{array}{cccccccc}
H_{n} & H_{n+1} & \cdots & H_{n+N-h-1} & H_{n+N-h+i_{h}} & \cdots & H_{n+N-2+i_{2}} & H_{n+N-1+i_{1}} \\
H_{n+1} & H_{n+2} & \cdots & H_{n+N-h} & H_{n+N-h+1+i_{h}} & \cdots & H_{n+N-1+i_{2}} & H_{n+N+i_{1}} \\
\vdots & \vdots & \cdots & \vdots & \vdots & \cdots & \vdots & \vdots \\
H_{n+N-1} & H_{N} & \cdots & H_{n+2 N-h-2} & H_{n+2 N-h-1+i_{h}} & \cdots & H_{n+2 N-3+i_{2}} & H_{n+2 N-2+i_{1}}
\end{array}\right| .
$$

It is possible to derive the bilinear equations from the Plücker relations which are identities between the determinants whose columns are shifted. In fact, Proposition 3.7 is obtained from the following identities:

LEMMA 3.11

$$
\tau_{N+1 \boxminus}^{n} \tau_{N}^{n}-\tau_{N+1 \square}^{n} \tau_{N \square}^{n}+\tau_{N+1}^{n} \tau_{N \square}^{n}=0
$$




$$
\begin{aligned}
& \tau_{N+1 \boxminus}^{n} \tau_{N}^{n}-\tau_{N+1 \square}^{n} \tau_{N \square}^{n}+\tau_{N+1}^{n} \tau_{N \amalg}^{n}=0, \\
& \tau_{N+1}^{n} \tau_{N \square \square}^{n}-\tau_{N+1 \square}^{n} \tau_{N \square}^{n}+\tau_{N}^{n} \tau_{N+1 \boxminus \square}^{n}=0 \text {, } \\
& \tau_{N+1 \square}^{n} \tau_{N \varpi}^{n}-\tau_{N+1 \varpi}^{n} \tau_{N \square}^{n}+\tau_{N}^{n} \tau_{N+1 \boxplus}^{n}=0 .
\end{aligned}
$$

Proposition 3.8 is derived from the same identity in $\hat{\tau}_{N}^{n}$. Similarly, we get Proposition 3.9 from the identities,

LEMMA 3.12

$$
\begin{gathered}
\tau_{N+1 \boxminus}^{n} \tau_{N}^{n+1}-\tau_{N+1 \square}^{n} \tau_{N}^{n+1}+\tau_{N+1}^{n} \tau_{N}^{n+1}=0, \\
\tau_{N+1 \boxminus}^{n} \tau_{N}^{n+1}-\tau_{N+1 \square}^{n} \tau_{N}^{n+1}+\tau_{N+1}^{n} \tau_{N}^{n+1}{ }_{\square}=0 . \\
\tau_{N+1}^{n} \tau_{N}^{n+1}-\tau_{N+1 \square}^{n} \tau_{N}^{n+1}{ }_{\square}+\tau_{N}^{n} \tau_{N+1 \boxminus}^{n+1}=0, \\
\tau_{N+1 \square}^{n} \tau_{N}^{n+1}-\tau_{N+1 \square}^{n} \tau_{N}^{n+1}{ }_{\square}+\tau_{N}^{n+1} \tau_{N+1}^{n} \boxminus=0 .
\end{gathered}
$$

We give the derivations of Lemmas 3.11 and 3.12 in the appendix.

We next construct the shift operators which are differential operators generating $\tau_{N Y}^{n}$ from $\tau_{N}^{n}$ by using the technique developed in refs. 20 and [5]. In fact, we have:

LEMMA 3.13

$$
\tau_{N \square}^{n}=\left(-\frac{d}{d x}+x N\right) \tau_{N}^{n} .
$$

Proof. Notice that $\tau_{N \square}^{n}$ is expressed by

$$
\tau_{N \square}^{n}=\left(\begin{array}{cccc}
H_{n+1} & H_{n+2} & \cdots & H_{n+N} \\
H_{n+2} & H_{n+3} & \cdots & H_{n+N+1} \\
\vdots & \vdots & \ddots & \vdots \\
H_{n+N} & H_{n+N+1} & \cdots & H_{n+2 N-1}
\end{array}\right) \cdot\left(\begin{array}{cccc}
\Delta_{11} & \Delta_{12} & \cdots & \Delta_{1 N} \\
\Delta_{21} & \Delta_{22} & \cdots & \Delta_{2 N} \\
\vdots & \vdots & \ddots & \vdots \\
\Delta_{N 1} & \Delta_{N 2} & \cdots & \Delta_{N N}
\end{array}\right),
$$

where $\Delta_{i j}$ is the $(i, j)$-cofactor of $\tau_{N}^{n}$ and $A \cdot B$ denotes a standard scalar product for $N \times N$ matrices $A=\left(a_{i j}\right)$ and $B=\left(b_{i j}\right)$ which is defined as

$$
A \cdot B=\sum_{i, j=1}^{N} a_{i j} b_{i j}=\operatorname{Tr} A^{t} B .
$$

The first matrix of (91) is rewritten by using the recursion relation (65) as

$$
\begin{array}{r}
-\left(\begin{array}{cccc}
\partial_{x} H_{n} & \partial_{x} H_{n+1} & \cdots & \partial_{x} H_{n+N-1} \\
\partial_{x} H_{n+1} & \partial_{x} H_{n+2} & \cdots & \partial_{x} H_{n+N} \\
\vdots & \vdots & \ddots & \vdots \\
\partial_{x} H_{n+N-1} & \partial_{x} H_{n+N} & \cdots & \partial_{x} H_{n+2 N-2}
\end{array}\right) \\
+x\left(\begin{array}{cccc}
H_{n} & H_{n+1} & \cdots & H_{n+N-1} \\
H_{n+1} & H_{n+2} & \cdots & H_{n+N} \\
\vdots & \vdots & \ddots & \vdots \\
H_{n+N-1} & H_{n+N} & \cdots & H_{n+2 N-2}
\end{array}\right)
\end{array}
$$


Then applying the dot product to eq.(93), we get

$$
\tau_{N \square}^{n}=\left(-\frac{d}{d x}+x N\right) \tau_{N}^{n} .
$$

Thus we have proved Lemma 3.13 .

For the shift operators of second order, we have:

LEMMA 3.14

$$
\begin{aligned}
& \tau_{N \square}^{n}=\frac{1}{2}\left(\frac{d^{2}}{d x^{2}}-(2 N+1) x \frac{d}{d x}+x^{2} N(N-1)-N(N+n+1)\right) \tau_{N}^{n}, \\
& \tau_{N \boxminus}^{n}=\frac{1}{2}\left(\frac{d^{2}}{d x^{2}}-(2 N-1) x \frac{d}{d x}+N(N-1) x^{2}+N(N+n-1)\right) \tau_{N}^{n} .
\end{aligned}
$$

Proof. We consider

$$
\begin{gathered}
\tau_{N \square}^{n}+\tau_{N \boxminus}^{n}=\left(\begin{array}{ccccc}
H_{n+1} & H_{n+2} & \cdots & H_{n+N-1} & H_{n+N+1} \\
H_{n+2} & H_{n+3} & \cdots & H_{n+N} & H_{n+N+2} \\
\vdots & \vdots & \vdots & \vdots & \vdots \\
H_{n+N} & H_{n+N+1} & \cdots & H_{n+2 N-2} & H_{n+2 N}
\end{array}\right) \\
\cdot\left(\begin{array}{cccc}
\Delta_{\square 11} & \Delta_{\square 12} & \cdots & \Delta_{\square 1 N} \\
\Delta_{\square 21} & \Delta_{\square 22} & \cdots & \Delta_{\square 2 N} \\
\vdots & \vdots & \ddots & \vdots \\
\Delta_{\square N 1} & \Delta_{\square N 2} & \cdots & \Delta_{\square N N}
\end{array}\right),
\end{gathered}
$$

where $\Delta_{\square i j}$ is $(i, j)$ cofactor of $\tau_{N \square}$. The first matrix in the right-hand side is equal to

$$
\begin{array}{r} 
\\
-\left(\begin{array}{ccccc}
\partial_{x} H_{n} & \partial_{x} H_{n+1} & \cdots & \partial_{x} H_{n+N-2} & \partial_{x} H_{n+N} \\
\partial_{x} H_{n+1} & \partial_{x} H_{n+2} & \cdots & \partial_{x} H_{n+N-1} & \partial_{x} H_{n+N+1} \\
\vdots & \vdots & \vdots & \vdots & \vdots \\
\partial_{x} H_{n+N-1} & \partial_{x} H_{n+N} & \cdots & \partial_{x} H_{n+2 N-1} & \partial_{x} H_{n+2 N-1}
\end{array}\right) \\
+x\left(\begin{array}{ccccc}
H_{n} & H_{n+1} & \cdots & H_{n+N-2} & H_{n+N} \\
H_{n+1} & H_{n+2} & \cdots & H_{n+N-1} & H_{n+N+1} \\
\vdots & \vdots & \vdots & \vdots & \vdots \\
H_{n+N-1} & H_{n+N} & \cdots & H_{n+2 N-1} & H_{n+2 N-1}
\end{array}\right),
\end{array}
$$

Applying the dot product to eq.(98), we obtain,

$$
\begin{aligned}
\tau_{N \square}^{n}+\tau_{N \boxminus}^{n} & =\left(-\frac{d}{d x}+x N\right) \tau_{N \square}^{n} \\
& =\left(\frac{d^{2}}{d x^{2}}-2 x N \frac{d}{d x}-N+x^{2} N^{2}\right) \tau_{N}^{n} .
\end{aligned}
$$


Next we consider the following equality,

$$
\tau_{N \square}^{n}-\tau_{N \boxminus}^{n}=\left(\begin{array}{cccc}
H_{n+2} & H_{n+3} & \cdots & H_{n+N+1} \\
H_{n+3} & H_{n+4} & \cdots & H_{n+N+2} \\
\vdots & \vdots & \ddots & \vdots \\
H_{n+N+1} & H_{n+N+2} & \cdots & H_{n+2 N}
\end{array}\right) \cdot\left(\begin{array}{cccc}
\Delta_{11} & \Delta_{12} & \cdots & \Delta_{1 N} \\
\Delta_{21} & \Delta_{22} & \cdots & \Delta_{2 N} \\
\vdots & \vdots & \ddots & \vdots \\
\Delta_{N 1} & \Delta_{N 2} & \cdots & \Delta_{N N}
\end{array}\right) .
$$

The first matrix of the right hand side of $(100)$ is rewritten as

$$
\begin{gathered}
-\left(\begin{array}{cccc}
\partial_{x} H_{n+1} & \partial_{x} H_{n+2} & \cdots & \partial_{x} H_{n+N} \\
\partial_{x} H_{n+2} & \partial_{x} H_{n+3} & \cdots & \partial_{x} H_{n+N+1} \\
\vdots & \vdots & \ddots & \vdots \\
\partial_{x} H_{n+N} & \partial_{x} H_{n+N+1} & \cdots & \partial_{x} H_{n+2 N-1}
\end{array}\right) \\
+x\left(\begin{array}{cccc}
H_{n+1} & H_{n+2} & \cdots & H_{n+N} \\
H_{n+2} & H_{n+3} & \cdots & H_{n+N+1} \\
\vdots & \vdots & \ddots & \vdots \\
H_{n+N} & H_{n+N+1} & \cdots & H_{n+2 N-1}
\end{array}\right)
\end{gathered}
$$

The first matrix of eq.(101) is rewritten as

$$
\begin{aligned}
& \left(\begin{array}{cccc}
(n+1) H_{n} & (n+2) H_{n+1} & \cdots & (n+N) H_{n+N-1} \\
(n+2) H_{n+1} & (n+3) H_{n+2} & \cdots & (n+N+1) H_{n+N} \\
\vdots & \vdots & \ddots & \vdots \\
(n+N) H_{n+N-1} & (n+N+1) H_{n+N} & \cdots & (n+2 N-1) H_{n+2 N-2}
\end{array}\right) \\
& =\left(\begin{array}{cccc}
(n+1) H_{n} & (n+1) H_{n+1} & \cdots & (n+1) H_{n+N-1} \\
(n+2) H_{n+1} & (n+2) H_{n+2} & \cdots & (n+2) H_{n+N} \\
\vdots & \vdots & \ddots & \vdots \\
(n+N) H_{n+N-1} & (n+N) H_{n+N} & \cdots & (n+N) H_{n+2 N-2}
\end{array}\right) \\
& +\left(\begin{array}{cccc}
0 & H_{n+1} & \cdots & (N-1) H_{n+N-1} \\
0 & H_{n+2} & \cdots & (N-1) H_{n+N} \\
\vdots & \vdots & \ddots & \vdots \\
0 & H_{n+N} & \cdots & (N-1) H_{n+2 N-2}
\end{array}\right)
\end{aligned}
$$

Applying the dot product to eq.(102), we get

$$
[\{(n+1)+\cdots+(n+N)\}+1+\cdots+(N-1)] \tau_{N}^{n}=N(N+n) \tau_{N}^{n}
$$

Moreover, from the second term of eq.(101), we have

$$
x \tau_{N \square}=x\left(-\frac{d}{d x}+x N\right) \tau_{N}^{n} .
$$

Finally, we obtain

$$
\tau_{N \square}^{n}-\tau_{N \boxminus}^{n}=\left(-x \frac{d}{d x}+x^{2} N-N(N+n)\right) \tau_{N}^{n} .
$$


From eqs.(99) and (103), we obtain eqs. (95) and (96). Thus we have proved Lemma 3.14.

Continuing the similar but tedious calculations, we get the following shift operators of third order:

LEMMA 3.15

$$
\begin{aligned}
\tau_{N \amalg}^{n} & =\frac{1}{6}\left[-\frac{d^{3}}{d x^{3}}+3(N+1) x \frac{d^{2}}{d x^{2}}+\left(-\left(3 N^{2}+6 N+2\right) x^{2}+3 N^{2}+3 n N+7 N+2 n+3\right) \frac{d}{d x}\right. \\
& \left.+N x\left((N+1)(N+2) x^{2}-3 n N-3 N^{2}-4 n-9 N-6\right)\right] \tau_{N}^{n}, \\
\tau_{N \boxminus}^{n} & =\frac{1}{3}\left[-\frac{d^{3}}{d x^{3}}+3 N x \frac{d^{2}}{d x^{2}}+\left(\left(-3 N^{2}+1\right) x^{2}-n+N\right) \frac{d}{d x}+N x\left(\left(N^{2}-1\right) x^{2}+2 n\right)\right] \tau_{N}^{n}(105) \\
\tau_{N \boxminus}^{n} & =\frac{1}{6}\left[-\frac{d^{3}}{d x^{3}}+3 x(N-1) \frac{d^{2}}{d x^{2}}+\left(\left(-3 N^{2}+6 N-2\right) x^{2}-3 n N+2 n-3 N^{2}+7 N-3\right) \frac{d}{d x}\right. \\
& \left.+N x\left(\left(N^{2}-3 N+2\right) x^{2}+3 n N-4 n+3 N^{2}-9 N+6\right)\right] \tau_{N}^{n} .
\end{aligned}
$$

Now we are ready to prove the Proposition 3.7. Substituting the shift operators in Lemmas 3.13 and 3.14 into the identities (82) and (83) in Lemma 3.11, we get eqs. (72) and (73). Equation (74) is obtained in similar manner by using the shift operators of fourth order, which will be given in the appendix. This completes the proof of Proposition 3.7. Proposition 3.9 is

proved from the identities in Lemma 3.12. Finally, to prove Proposition 3.8, it is necessary to calculate the shift operators for $\hat{\tau}_{N}^{n}$. We omit the detailes, but this is done just by replacing the recursion relations eq.(65) by

$$
\frac{d}{d x} \hat{H}_{n}+x \hat{H}_{n}=\hat{H}_{n+1}, \quad \frac{d}{d x} \hat{H}_{n}=n \hat{H}_{n-1} .
$$

Thus proof of Theorems 3.2 and 3.5 is completed.

\section{Concluding Remarks}

In this article, we have investigated the hierarchies of rational solutions for $\mathrm{P}_{\mathrm{IV}}$, and shown that

1. Solutions in $-2 / 3 z$ hierarchy are expressed in terms of 3-reduced Schur functions. In particular, the Okamoto polynomials are nothing but their special cases.

2. Solutions in $-2 z$ and $-1 / z$ hierarchies are expressed in terms of Casorati determinant of the Hermite polynomials. Moreover, they are also expressed by special cases of the Schur functions. 
It might be an important and interesting problem to characterize the non-classical polynomials which appears in the algebraic solutions of the Painlevé equations listed in 2] by studying the determinant expressions with the aid of results of the soliton theory.

We finally note that after obtaining the results, the authors were informed that Noumi and Yamada have independently obtained the Schur function expression for the rational solutions of $\mathrm{P}_{\mathrm{IV}}[21$.

\section{Acknowledgement}

The authors are grateful to Professors B. Grammaticos, A. Ramani and J. Hietarinta for discussions and encouragement. They also thank Professors H. Umemura and K. Okamoto for their interests in this work and discussions. One of the authors(K.K) was supported by the Grantin-aid for Encouragement of Young Scientist, The Ministry of Education, Science, Sports and Culture of Japan, No. 09740164.

\section{A Derivation of Plücker Relations}

We consider the following identity of $(2 N+2) \times(2 N+2)$ determinant,

$$
0=\left|\begin{array}{rrrr:cc|ccc}
0 & 1 & \cdots & N-1 & & \varnothing & N & N+1 & \phi_{1} \\
\hdashline & \varnothing & & 0 & \cdots & N-2 & N & N+1 & \phi_{1}
\end{array}\right|,
$$

where " $k$ " denotes the column vector,

$$
" k "=\left(\begin{array}{c}
H_{n+k} \\
H_{n+k+1} \\
\vdots \\
H_{n+k+N-1}
\end{array}\right),
$$

and

$$
\phi_{1}=\left(\begin{array}{c}
0 \\
0 \\
\vdots \\
1
\end{array}\right) \text {. }
$$

Applying the Laplace expansion on the right hand side of eq.(108), we get

$$
\begin{aligned}
0 & =|0, \cdots, N-2, N-1, N| \times\left|0, \cdots N-2, N+1, \phi_{1}\right| \\
& -|0, \cdots, N-2, N-1, N+1| \times\left|0, \cdots N-2, N, \phi_{1}\right| \\
& +\left|0, \cdots, N-2, N-1, \phi_{1}\right| \times|0, \cdots N-2, N, N+1| \\
& =\tau_{N+1}^{n} \tau_{N \square}^{n}-\tau_{N+1 \square}^{n} \tau_{N \square}^{n}+\tau_{N}^{n} \tau_{N+1 \boxminus}^{n}
\end{aligned}
$$


which is nothing but eq. (82). Similarly, eq.(83) is derived from the following identity,

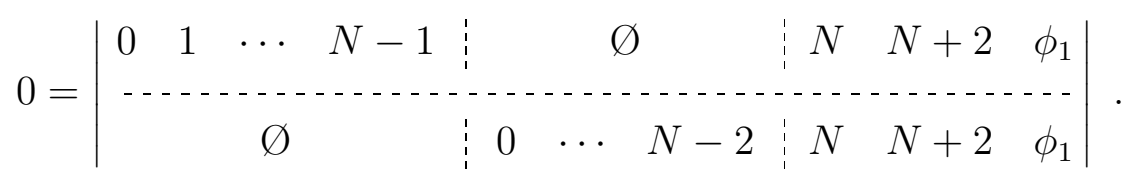

Moreover, we have the following higher order identities:

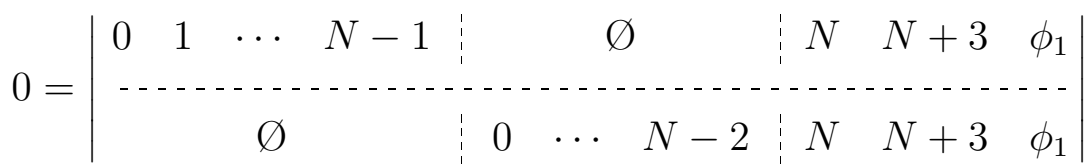

$$
\begin{aligned}
& =|0, \cdots, N-2, N-1, N| \times\left|0, \cdots N-2, N+3, \phi_{1}\right| \\
& -|0, \cdots, N-2, N-1, N+3| \times\left|0, \cdots N-2, N, \phi_{1}\right| \\
& +\left|0, \cdots, N-2, N-1, \phi_{1}\right| \times|0, \cdots N-2, N, N+3| \\
& =\tau_{N+1}^{n} \tau_{N \amalg \square}^{n}-\tau_{N+1 \varpi \square}^{n} \tau_{N \square}^{n}+\tau_{N}^{n} \tau_{N+1 \varpi}^{n},
\end{aligned}
$$

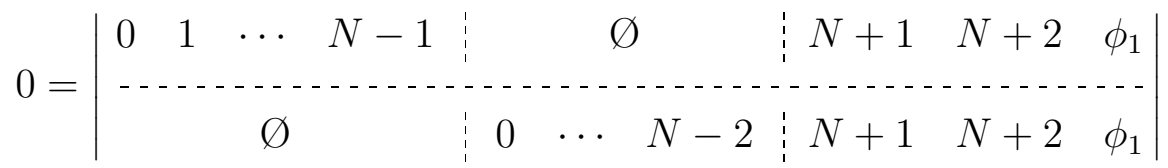

$$
\begin{aligned}
& =|0, \cdots, N-2, N-1, N+1| \times\left|0, \cdots N-2, N+2, \phi_{1}\right| \\
& -|0, \cdots, N-2, N-1, N+2| \times\left|0, \cdots N-2, N+1, \phi_{1}\right| \\
& +\left|0, \cdots, N-2, N-1, \phi_{1}\right| \times|0, \cdots N-2, N+1, N+2| \\
& =\tau_{N+1 \square}^{n} \tau_{N \square}^{n}-\tau_{N+1 \square}^{n} \tau_{N \square}^{n}+\tau_{N}^{n} \tau_{N+1 \boxplus}^{n} \cdot
\end{aligned}
$$

Identities between $\tau_{N+1 Y}^{n}$ and $\tau_{N Y}^{n+1}$ are derived only by replacing $\phi_{1}$ in the above identities by

$$
\phi_{2}=\left(\begin{array}{c}
1 \\
0 \\
\vdots \\
0
\end{array}\right)
$$

\section{B List of the Shift Operators}

$$
\begin{aligned}
\tau_{N \amalg}^{n} & =\frac{1}{24}\left[\frac{d^{4}}{d x^{4}}-2(2 N+3) x \frac{d^{3}}{d x^{3}}+\left\{\left(6 N^{2}+18 N+11\right) x^{2}-6 n N-8 n-6 N^{2}-22 N-18\right\} \frac{d^{2}}{d x^{2}}\right. \\
& +x\left\{\left(-4 N^{3}-18 N^{2}-22 N-6\right) x^{2}+12 N^{3}+12 n N^{2}+58 N^{2}+30 n N+12 n+78 N+25\right\} \frac{d}{d x} \\
& +N\left\{\left(N^{3}+6 N^{2}+11 N+6\right) x^{4}-\left(6 n N^{2}+22 n N+18 n+6 N^{3}+36 N^{2}+66 N+36\right) x^{2}\right. \\
& \left.\left.+3 N^{3}+3 n^{2} N+6 n N^{2}+18 N^{2}+6 n^{2}+24 n N+22 n+33 N+18\right\}\right] \tau_{N}^{n},
\end{aligned}
$$




$$
\begin{aligned}
& \tau_{N \boxminus \square}^{n}=\frac{1}{8}\left[\frac{d^{4}}{d x^{4}}-2 x(2 N+1) \frac{d^{3}}{d x^{3}}+\left\{\left(6 N^{2}+6 N-1\right) x^{2}-2 n N-2 N^{2}-6 N-2\right\} \frac{d^{2}}{d x^{2}}\right. \\
& +x\left\{\left(-4 N^{3}-6 N^{2}+2 N+2\right) x^{2}+4 n N^{2}-2 n N-4 n+4 N^{3}+10 N^{2}+2 N-3\right\} \frac{d}{d x} \\
& +N\left\{\left(N^{3}+2 N^{2}-N-2\right) x^{4}+\left(-2 N^{3}-4 N^{2}-2 n N^{2}+2 n N+6 n+2 N+4\right) x^{2}\right. \\
& \left.\left.-N^{3}+n^{2} N-2 n N^{2}-2 N^{2}-2 n^{2}-4 n N-2 n+N+2\right\}\right] \tau_{N}^{n} \\
& \tau_{N \boxplus}^{n}=\frac{1}{12}\left[\frac{d^{4}}{d x^{4}}-4 N x \frac{d^{3}}{d x^{3}}+\left\{\left(6 N^{2}-1\right) x^{2}+4 n+2 N\right\} \frac{d^{2}}{d x^{2}}\right. \\
& +x\left\{2 N\left(-2 N^{2}+1\right) x^{2}-6 n N-2 N^{2}+1\right\} \frac{d}{d x} \\
& \left.+N\left\{N\left(N^{2}-1\right) x^{4}+2 n N x^{2}+3 N^{3}+3 n^{2} N+6 n N^{2}-2 n-3 N\right\}\right] \tau_{N}^{n}, \\
& \tau_{N \boxminus}^{n}=\frac{1}{8}\left[\frac{d^{4}}{d x^{4}}+2 x(-2 N+1) \frac{d^{3}}{d x^{3}}+\left\{\left(6 N^{2}-6 N-1\right) x^{2}+2 n N+2 N^{2}-6 N+2\right\} \frac{d^{2}}{d x^{2}}\right. \\
& +x\left\{\left(-4 N^{3}+6 N^{2}+2 N-2\right) x^{2}-4 N^{3}-4 n N^{2}+10 N^{2}-2 n N+4 n-2 N-3\right\} \frac{d}{d x} \\
& +\left\{\left(N^{3}-2 N^{2}-N+2\right) x^{4}+\left(2 n N^{2}+2 n N-6 n+2 N^{3}-4 N^{2}-2 N+4\right) x^{2}\right. \\
& \left.\left.-n^{2} N+2 n^{2}-2 n N^{2}+4 n N-2 n-N^{3}+2 N^{2}+N-2\right\}\right] \tau_{N}^{n}, \\
& \tau_{N 日}^{n}=\frac{1}{24}\left[\frac{d^{4}}{d x^{4}}+2 x(-2 N+3) \frac{d^{3}}{d x^{3}}+\left\{\left(6 N^{2}-18 N+11\right) x^{2}+6 N^{2}+6 n N-22 N-8 n+18\right\} \frac{d^{2}}{d x^{2}}\right. \\
& +x\left\{\left(-4 N^{3}+18 N^{2}-22 N+6\right) x^{2}-12 N^{3}-12 n N^{2}+58 N^{2}+30 n N-12 n-78 N+25\right\} \frac{d}{d x} \\
& +N\left\{\left(N^{3}-6 N^{2}+11 N-6\right) x^{4}+\left(6 N^{3}+6 n N^{2}-36 N^{2}-22 n N+18 n+66 N-36\right) x^{2}\right. \\
& \left.\left.+3 N^{3}+3 n^{2} N+6 n N^{2}-18 N^{2}-6 n^{2}-24 n N+22 n+33 N-18\right\}\right] \tau_{N}^{n} .
\end{aligned}
$$

\section{References}

[1] See, for example, Painlevé Transcendents: Their Asymptotics and Physical Applications eds. by D. Levi and P. Winternitz (NATO ASI series B278, Prenum, New York, 1992), 175.

[2] H. Umemura, "Special Polynomials Assciated with the Painlevé Equations I", preprint.

[3] A. I. Yablonskii, Vesti A.N.BSSR., Ser. Fiz-Tekh. Nauk. 3(1959), 30. (in Russian)

[4] A. P. Vorobe'v, Diff. Uravn. 1(1965), 58.

[5] K. Kajiwara and Y. Ohta, J. Math. Phys. 37(1996), 4693. 
[6] A. P. Bassom, P. A. Clarkson and A. C. Hicks, Stud. Appl. Math. 95(1995), 1

[7] Y. Murata, Funkcial. Ekvac., 28(1985), 1.

[8] A. S. Fokas, U. Mugan and M. J. Ablowitz, Physica 30D(1988), 243.

[9] V. I. Gromak, Diff. Eq. 23(1987), 506.

[10] A. P. Bassom and C. A. Clarkson, Phys. Lett. A194(1994), 358.

[11] N. A. Lukashevich, Diff. Eq. 3(1967), 395.

[12] K. Okamoto, Math. Ann. 275(1986), 221.

[13] P. A. Clarkson, J. Phys. A 22(1989), 2355.

[14] G. R. W. Quispel, F. W. Nijhoff and H. W. Capel, Phys. Lett. A 91(1982), 143.

[15] S. Kawamoto, J. Phys. Soc. Jpn., 53(1984), 2922.

[16] T. Nishitani and M. Tajiri, Phys. Lett. 89A (1982), 379.

[17] M. Jimbo and T. Miwa, Publ. RIMS, Kyoto Univ. 19(1983), 943.

[18] H. Umemura and H. Watanabe, "Solutions of the second and fourth Painlevé equations I $"$, to appear in Nagoya Math. J.

[19] R. Hirota and J. Satsuma, Prog. Theor. Phys. Suppl.59(1976), 64.

[20] Y. Ohta and A. Nakamura, J. Phys. Soc. Jpn., 61(1992), 4295.

[21] M. Noumi and Y. Yamada, "Symmetries in the fourth Painlevé equation and Okamoto polynomials", preprint, q-alg/9708018. 